University of Wollongong

Research Online

Faculty of Engineering and Information

Faculty of Engineering and Information

Sciences - Papers: Part A

Sciences

$1-1-2015$

\title{
Evaluating distributed generations in utility operation and planning issues using a novel fusion PSO-SFLA algorithm
}

\author{
Esmaeil Mahboubi-Moghaddam \\ Shiraz University of Technology \\ Jamshid Aghaei \\ Shiraz University of Technology, jamshid@uow.edu.au \\ Kashem M. Muttaqi \\ University of Wollongong, kashem@uow.edu.au \\ Behrouz Zoghdar-Moghadam-Shahrekohne \\ Shiraz University of Technology \\ Mohammad Rasoul Narimani \\ Missouri University of Science and Technology
}

Follow this and additional works at: https://ro.uow.edu.au/eispapers

Part of the Engineering Commons, and the Science and Technology Studies Commons 


\title{
Evaluating distributed generations in utility operation and planning issues using a novel fusion PSO-SFLA algorithm
}

\author{
Abstract \\ Increasing demand of electrical energy has leaded to utilization of more and more Distributed generation \\ (DG) sources in distribution systems. Since the locations and capacities of the DG sources connected to \\ the distribution system profoundly impact on reducing system loss and improving system reliability, so \\ placement and sizing indication of DGs is the most substantial process in distribution systems. By adding \\ the reliability objective to this problem, it becomes more complicated than before and it needs to be \\ solved with an accurate algorithm. To this reason, to solve the proposed problem a new approach based \\ on the mixture of two algorithms named as Particle Swarm Optimization (PSO) and the Shuffle Frog \\ Leaping algorithm (SFLA) is applied in this paper. A meticulous performance analysis is fulfilled on a \\ 33-bus system in order to demonstrate the effectiveness of the presented methodology.

\section{Keywords} \\ pso, sfla, algorithm, distributed, generations, evaluating, utility, operation, planning, issues, novel, fusion \\ Disciplines \\ Engineering | Science and Technology Studies

\section{Publication Details} \\ E. Mahboubi-Moghaddam, J. Aghaei, K. M. Muttaqi, B. Zoghdar-Moghadam-Shahrekohne \& M. Rasoul. \\ Narimani, "Evaluating distributed generations in utility operation and planning issues using a novel fusion \\ PSO-SFLA algorithm," in Power Engineering Conference (AUPEC), 2015 Australasian Universities, 2015, \\ pp. 1-6.
}




\title{
Evaluating Distributed Generations in Utility Operation and Planning Issues Using a Novel Fusion PSO-SFLA Algorithm
}

\author{
Esmaeil Mahboubi-Moghaddam ${ }^{1}$, Jamshid Aghaei ${ }^{1}$, Kashem M. Muttaqi ${ }^{2}$, \\ Behrouz Zoghdar-Moghadam-Shahrekohne ${ }^{1}$, Mohammad Rasoul Narimani ${ }^{3}$
}

1. Department of Electrical and Electronics Engineering, Shiraz University of Technology, Shiraz, Iran

2. Australian Power Quality and Reliability Center (APQRC), School of Electrical, Computer and Telecommunications Engineering (SECTE), University of Wollongong, Wollongong, NSW 2522, Australia

3. PhD Student in Electrical Engineering, Missouri University of Science and Technology

E-mails: e.mahboubi@ sutech.ac.ir, aghaei@sutech.ac.ir, kashem@uow.edu.au,

\begin{abstract}
Increasing demand of electrical energy has leaded to utilization of more and more Distributed generation (DG) sources in distribution systems. Since the locations and capacities of the DG sources connected to the distribution system profoundly impact on reducing system loss and improving system reliability, so placement and sizing indication of DGs is the most substantial process in distribution systems. By adding the reliability objective to this problem, it becomes more complicated than before and it needs to be solved with an accurate algorithm. To this reason, to solve the proposed problem a new approach based on the mixture of two algorithms named as Particle Swarm Optimization (PSO) and the Shuffle Frog Leaping algorithm (SFLA) is applied in this paper. A meticulous performance analysis is fulfilled on a 33-bus system in order to demonstrate the effectiveness of the presented methodology.
\end{abstract}

Keywords-component; Distributed Generation, Reliability, Particle Swarm Optimization, Shuffle Frog Leaping Algorithm

\section{INTRODUCTION}

In the local distribution systems the newly installed distributed or decentralized generation units may not be dispatched by a central operator. Over the recent years, the interest for using DG sources has been amplified by both the consumer and distribution companies due to issues such as the extensive advances in several generation technologies, deregulation of power systems, environmental impacts of the electric power generation and tense constraints construction of new transmission lines for long distance power transmission. As mentioned before the locations and capacities of DG sources connected to the distribution system have a profound effect on their application. Correctly placement of DGs in a network may have some profits including loss reduction, peak shaving, voltage control, ancillary services, higher power quality and reliability indices, deferral of transmission and distribution systems reinforcement, and environmental concerns may be achieved [1]. To this reason, consideration of some aspects in optimization problem for optimal sitting and sizing of DGs is of great importance. To deal with this problem numerous extraordinary works have been carried out. Different methods such as analytical procedures in addition to deterministic and heuristic methods are presented by many researches in order to solve the problem. It is noteworthy to say that the main dissimilarities among these studies refer to the formulation, solution methodology and assumptions of the problem. During recent years, it has been discovered by study of evolutionary algorithms that better results can be obtained by means of resourcefully applying these methods. Over the recent few years several complex optimization problems such as estimate energy demand problem [2], power system planning problem [2] and the OPF problem [2] are solved by utilization of the newly presented evolutionary optimization algorithms. In $[3,4]$ the authors only surveyed the optimum DG placement in the distribution system. A hybrid PSO and genetic algorithm is proposed in [5], to solve a multi-objective index including voltage profile and stability as well as power losses. In [6], a Pareto-based multi-objective placement and sizing of multiple DGs have been obtained using an improved honey bee mating optimization (HBMO). The advantage of the presented approach with respect to others presented in literatures is that this approach considers one of the most significant reliability indices called Energy Not Supplied (ENS) which plays a main role in reliability of distribution systems. Also, the solution methodology that has been utilized in this approach has supremacy over those reported in literatures.

There are many formulations in relation to power system reliability and availability, one of them is Energy Not Supplied which is considered as an objective function in this approach. Encouraging network owners to plan, operate and maintain their networks in an optimal socio-economic manner is the principal objective, since they have to pay fine for energy not supplied hours, and thereby provide a socioeconomic optimal level of reliability. Furthermore with expansion of power distribution network and increase of power electricity demand, the distribution loss in power system becomes a crucial problem. Hence it is important to keep the amount of the distribution loss in an acceptable range or reduce it. To this end power loss has been considered as an objective function in this approach. 
A multi-objective method is required in order to solve the presented problem, since it is a Multi-objective Optimization Problem (MOP). Thus, a Pareto-based approach is utilized in this paper which can achieve a set of optimal solutions instead of one. Distribution network operators can select one of these optimal solutions in different situations regarding their experience. Meanwhile, in this paper, a fuzzy decision making tools is applied to find the best compromise solution among all the Pareto optimal solutions. Besides the importance of multi objective solution methodology, the optimization algorithm that has been utilized for solving the optimization problem plays an effective role as well. Particle Swarm Optimization (PSO) algorithm is one of the most accurate and trustworthy methods which has validated its astonishing abilities in solving optimization problems among all of the evolutionary algorithms and has been applied to variety of the optimization problems [2]. In finding appropriate solution in optimization problem in addition to PSO algorithm, the Shuffle Frog Leaping Algorithm (SFLA) has verified its capabilities [2]. But there are some drawbacks besides numerous privileges of the aforementioned algorithms like their converging into the global optima in long period of time or their trapping in local optima. It is worthwhile to note some particular characteristics of evolutionary algorithm, having a strong global search and local search are the specific characteristics of PSO and SFLA algorithms, respectively. Thus, the best way is applying both algorithms for achieving some better research characteristics, which is strongly suggested and exactly defined in this paper. To this end this paper presents a Fusion PSO-SFLA (FPSOSFLA) technique, which profits from the privileges of both PSO and SFLA algorithms, in order to cope with the complexity of the proposed problem.

The suitability of the projected algorithm is demonstrated by applying it to a 33-bus IEEE test system. The capability of the proposed algorithm in finding global optima in the presented optimization problem can be verified by usage of Simulation results.

The main contributions of this approach are:

- Proposing a Fusion PSO-SFLA (FPSO-SFLA) technique.

- Considering the reliability (Energy Not Supplied (ENS)) objective in the proposed problem

- Considering the effects of DGs on different objective functions

\section{PROBLEM FORMULATION}

The considered objective functions and constraints in the proposed work are as follows:

A. Objective functions

- Loss [7]

$f_{1}(X)=\sum_{i=1}^{N_{\text {Bnanch }}} R_{i} \times\left|I_{i}\right|^{2}$

Where $R$ and $I_{i}$ are resistance and actual current of the $i^{\text {th }}$ branch, respectively and $N_{\text {Branch }}$ is the number of branches.
Since this objective function has not been considered too much in literatures, it is scrutinized in this section. To this end consider a distribution network with $n$ nodes in which $n>1$ and consider node 0 as the source of this network.

Assume that all nodes except the source have an active power $P_{i}[\mathrm{~kW}], i \in\{1,2, \ldots, n-1\}$. The objective function's value at each node can be calculated in terms of the reliability parameters of the distribution network [8]. In this regard, a distribution branch between nodes $i$ and $j$ is associated with the following parameters: a failure rate $\lambda_{i j}$ ([fail $\left.\left./ \mathrm{km}-\mathrm{yr}\right]\right)$, an average reparation time $t_{i, j}([\mathrm{~h} / \mathrm{fail}])$, and a line length $d_{i, j}$ ([km]). The reparation time is the time which is elapsed to reestablish the service to a faulty zone after the failure has been fixed. Assume that every distribution branch incorporates a sectionalizing device on it in which, when a network reconfiguration process is triggered, such devices can be acted to change the network topology. In accordance with [8], the ENS at the node can be calculated as follows:

$$
E N S_{i}=P_{i} \sum_{i, j \in V, i \neq j}\left(U_{i, j}\right)
$$

Where $V=\{0,1, \ldots, n-1\}$ is the bunch of nodes in distribution network and $U_{j, i}$ is the service unavailability related to the reparation time of all the branches connected to the node $i$. In other word, $U_{j, i}$ is related to reparation time of all downstream branches of node $i$. It is worthwhile to note that the summation in (2) has to be understood as the sum of all the unavailability related to the $i^{\text {th }}$ node. The unavailability $U_{j, i}$ can be expressed as follows:

$U_{j, i}=\lambda_{j, i} \times d_{j, i} \times t_{j, i}$

In conclusion, the ENS of the whole distribution network is computed as the summation of all nodes except the node 0 , as follows:

$f_{2}(X)=E N S=\sum_{i=2}^{N_{B u s}} E N S_{i}$

\section{B. Constraints}

- Distribution line absolute power limits

$\left|P_{i j}^{\text {Line }}\right|<P_{i j, \text { Max }}^{\text {Line }}$

Where $P_{i j}^{\text {Line }}$ and $P_{i j, \text { Max }}^{\text {Line }}$ are the power flowing over the distribution branches and the maximum power transmitted between the nodes $i$ and $j$, respectively.

- Distribution power flow equations

$P_{i}=\sum_{i=1}^{N_{B u s}} V_{i} V_{j} Y_{i j} \cos \left(\theta_{i j}-\delta_{i}+\delta_{j}\right)$

$Q_{i}=\sum_{i=1}^{N_{B u s}} V_{i} V_{j} Y_{i j} \sin \left(\theta_{i j}-\delta_{i}+\delta_{j}\right)$

Where $P_{i}$ and $Q_{i}$ are the net injected active and reactive powers at the $i^{\text {th }}$ bus. $V_{i}$ and $\delta_{i}$ are the amplitude and the angle of the voltage at the $i^{t h}$ bus, respectively. Also, $Y_{i j}$ and 
$\theta_{i j}$ are the amplitude and the angle of the branch admittance between the $i^{\text {th }}$ and $j^{\text {th }}$ buses, respectively.

- Bus voltage limit

$V_{M \text { in }} \leq V_{i} \leq V_{\text {Max }}$

Where, $V_{M \text { in }}$ and $V_{M a x}$ are the minimum and maximum allowable voltage value of the $i^{\text {th }}$ node, respectively. Also, $V_{i}$ is the voltage magnitude of the $i^{\text {th }}$ node.

- Transformers limits

$\left|I_{t, i}\right| \leq I_{t, i}^{\operatorname{Max}} \quad i=1,2, \ldots ., N_{t}$

Where $I_{t, i}$ and $I_{t, i}^{\operatorname{Max}}$ are the current amplitude and its maximum allowable value of the $i^{\text {th }}$ transformer, respectively. $N_{t}$ is the number of transformers.

- Feeders limits

$\left|I_{f, i}\right| \leq I_{f, i}^{\text {Max }} \quad i=1,2, \ldots . ., N_{\text {Feeder }}$

Where, $I_{f, i}$ and $I_{f, i}^{\operatorname{Max}}$ are the current amplitude and its maximum allowable value of the $i^{\text {th }}$ feeder, respectively. $N_{\text {Feeder }}$ is the number of feeders.

Indeed, DGs in distribution networks can be modeled as PV or PQ models. DG can be controlled by two aforementioned models, since the distribution networks are unbalanced three phase systems [9], it is worthwhile to note that when DGs are considered as PV models, they must produce reactive power to keep voltage magnitudes in their proper boundaries. Some procedures regarding PV modeling of DGs can be found in the literature [9]. In this paper, in order to model DGs in distribution networks PQ model is employed.

\section{MULTI-OBJECTIVE SOLUTION STRATEGY}

MOP infers to providing of a set of solutions instead of one specific solution while multiple objectives in a simultaneous optimization conflict with each other. Formulation of MOP can be written as follow:

Minimize $F(\mathbf{X})=\left(F_{1}(\mathbf{X}), F_{2}(\mathbf{X}), \ldots \ldots ., F_{N_{o b j}}(\mathbf{X})\right)$

Subject to:

$u_{i}(\mathbf{X})<0$,

$$
\begin{aligned}
& i=1,2, \ldots \ldots \ldots, H \\
& i=1,2, \ldots \ldots \ldots, L
\end{aligned}
$$

Where, $F_{i}$ is the $i^{\text {th }}$ objective function, $\mathbf{X}$ is a determination vector that presents a solution, $N_{o b j}$ is the number of objectives. The number of the equality and the inequality constraints are $L$ and $H$, respectively.

\section{A. Normalizing the objective function}

To normalize the objective functions a fuzzy decision making function has been applied as follows:

$$
\mu_{i}=\left\{\begin{array}{lll}
1 & \text { if } & F_{i} \leq F_{i}^{\min } \\
\frac{F_{i}^{\max }-F_{i}}{F_{i}^{\max }-F_{i}^{\min }} & \text { if } & F_{i}^{\min }<F_{i}<F_{i}^{\max } i=1, \ldots, N_{o b j} \\
0 & \text { if } \quad F_{i} \geq F_{i}^{\max }
\end{array}\right.
$$

Where, $F_{i}^{\max }$ and $F_{i}^{\text {min }}$ are the acceptable maximum and minimum levels of $i^{\text {th }}$ objective function, respectively.

\section{B. Pareto optimal solution method}

The Pareto optimal solution method is used in order to attain a set of solutions for solving the MOP problem. By applying the dominance concept the Pareto method indicates the squad of solutions for multi-objective problems. The Pareto dominance for two decision vectors can be stated as follows [2]:

$$
\begin{aligned}
& \forall j \in\left\{1, \ldots, N_{o b j}\right\}, F_{j}\left(\mathbf{X}_{1}\right) \leq F_{j}\left(\mathbf{X}_{2}\right) \\
& \exists k \in\left\{1, \ldots, N_{o b j}\right\}, F_{k}\left(\mathbf{X}_{1}\right)<F_{k}\left(\mathbf{X}_{2}\right)
\end{aligned}
$$

All non-dominates solutions during all iterations of the optimization process are saved in a repository and sorted by decision making method, as stated before. Solutions with the highest value computed by equation (16) are assumed as the best compromise solution.

$$
N_{\mu}(j)=\frac{\sum_{k=1}^{N_{o b j}} \omega_{k} \times \mu_{k}(j)}{\sum_{j=1}^{m} \sum_{k=1}^{N_{o b j}} \omega_{k} \times \mu_{k}(j)}
$$

Where, $\omega_{k}$ is the weight factor for the $k^{\text {th }}$ objective function and $m$ is the number of the non-dominated solutions. The weight factor $\left(\omega_{k}\right)$ can be chosen by the operator due to the significance of the economic issues and the environmental allowance,. The solution with the maximum membership function $N_{\mu}$ is the most favored compromise solution based on the adopted weight factors and so is chosen as the best Pareto-optimal solution or the final solution of the problem.

\section{PROPOSED FUSION PSO-SFLA ALGORITHM}

\section{A. Particle Swarm Optimization}

An evolutionary optimization method motivated by social behavior of birds crowding or fish schooling is named as PSO [2]. A population is defined for the PSO algorithm which this population is continuously updating the searching space knowledge. The participating individuals of this population can be modeled as a particle that moves through the hyperspace and each one of these individuals represents a possible solution.

At each iteration $k$, each particle remembers its own best position associated with the best personal fitness value, Pbest $_{i}{ }^{k}$. The position with the best fitness value among all

Pbest $_{i}{ }^{k}$ is denoted by Gbest $^{k}$. Besides, the position and velocity of each particle are updated as follows:

$$
\begin{aligned}
& \mathbf{V}_{i}{ }^{k+1}=\omega \mathbf{V}_{i}{ }^{k}+C_{1} \text { rand }(.)_{1}\left(\mathbf{P b e s t}_{i}{ }^{k}-\mathbf{X}_{i}{ }^{k}\right) \\
& +C_{2} \operatorname{rand}(.)_{2}\left(\mathbf{G b e s t}^{k}-\mathbf{X}_{i}{ }^{k}\right) i=1, \ldots, N \\
& \mathbf{X}_{i}{ }^{k+1}=\mathbf{X}_{i}{ }^{k}+\mathbf{V}_{i}{ }^{k+1} \quad i=1, \ldots, N
\end{aligned}
$$


Where, $N$ is the number of the particles which constitute the population for the PSO algorithm. $\quad \mathbf{V}_{i}{ }^{k}$ and $\mathbf{V}_{i}{ }^{k+1}$ are the velocity of the $i^{\text {th }}$ particle at $k^{\text {th }}$ and $(k+1)^{\text {th }}$ iteration, respectively. $\omega$ is an inertia weight and $C_{1}, C_{2}$ are the positive coefficients selected between 0 and 2 that $C_{1}+C_{2} \leq 4$. $\operatorname{rand}(.)_{1}$ and $\operatorname{rand}(.)_{2}$ are the random numbers in the range 0 and 1 .

\section{B. Shuffle Frog Leaping Algorithm (SFLA)}

SFLA is a decrease based stochastic search method which begins with an initial population of frogs whose characteristics, known as memes, represent the decision variables. This algorithm includes elements of the local search and global information exchange [2]. The existing population in SFLA is divided into groups named as memeplexes which these groups search independently. The existing population of SFLA is divided into $q$ memeplexes and population regarding each of these memeplexes is $p$ frogs. In this process, the first frog goes to the first memeplex, the second frog goes to the second memeplex, frog $p$ goes to the $q^{\text {th }}$ memeplex, and $(p+1)^{\text {th }}$ frog goes back to the first memeplex, etc. In each memeplex, the frogs with the best and the worst fitness values are identified as $\mathbf{X}_{b}$ and $\mathbf{X}_{w}$, respectively. Also, the frog with the most qualified fitness level among all the memeplexes is identified as $\mathbf{X}_{g}$. Then, the following process is applied in order to improve only the frog with the worst fitness in each cycle. Accordingly, the position of the frog with the worst fitness is adjusted as follows:

$$
\begin{aligned}
& \text { Change in frog position } \\
& \left(C_{i}\right)=\operatorname{rand}(.)_{1}\left(\mathbf{X}_{b}-\mathbf{X}_{w}\right)+\operatorname{rand}(.)_{2}\left(\mathbf{X}_{g}-\mathbf{X}_{w}\right)
\end{aligned}
$$

New position $\mathbf{X}_{w}=$ current position of

$\mathbf{X}_{w}+\mathbf{C}_{i} ; \quad-\mathbf{C}_{\max } \leq \mathbf{C}_{i} \leq \mathbf{C}_{\max }$

Where, $\mathbf{C}_{\max }$ is the maximum allowed change in a frog position. If this process produces a better solution, it replaces the worst frog in each memeplex. If no improvement is achieved in this case, then a new population is randomly generated to replace that frog. The calculations then will continue for a specific number of iterations [2] and this procedure continues till the last iteration is accomplished.

\section{Fusion PSO-SFLA}

As mentioned before each algorithm has existing drawbacks, the PSO algorithm might be trapped in the local optima or converge to global optima in a long period of time. But for handling this problem this presented paper uses SFLA which has strong local search ability. In this regard, firstly $2 N$ population is generated and each algorithm starts its work with $N$ population. After each iterate, the best solutions of the both algorithms are compared with each other and one with the minimum value is selected as the best solution for both algorithms in the next iteration. Since the SFLA can amend the worst solutions in an effective way, worst solutions should be given to this algorithm and the best ones should be given to PSO algorithm.

\section{NUMERICAL RESULTS}

MATLAB programming codes for the FPSO-SFLA, distribution load flow algorithm and the proposed objectives functions are developed and incorporated for the simulation purposes in this paper. The performance and the advantages of the proposed algorithm are demonstrated on a widely referred system including 33-nodes. The rest of this approach is divided into two sections involving 33-node system with two and four distributed generations, respectively. The single line diagram of this test system is depicted in Fig 1. The essential data for 33-bus test system can be found [1]. Also, it is noteworthy to say that power nodes are presented in [1]. Furthermore, all the parameters related to ENS objective function have been considered typically in this study as follows $\lambda_{j, i}=0.065, d_{j, i}=1$ and $t_{j, i}=5$ for all branches.

\section{A. Section one: 33-node test system with two DGs}

This test system consists of two DGs. It should be noted that both DGs have a $1000 \mathrm{MW}$ capacity. In order to depict the effect of DGs on power loss objective function, Table I shows the obtained values related to both objectives for a 33-node distribution system with and without considering distributed generations. From this table it is clear that the power loss and ENS values are decreased drastically by adding DGs in distribution system. It means that DGs can play a powerful role in decreasing of the power loss in distribution systems. Also, the reliability metrics such as ENS, which are important characteristics in power system operation, usually are ignored in operation of distribution system problems. Therefore the most substantial contribution of this paper is devoted to consideration of ENS objective accompany with power loss objective function.

The convergence plot for loss and ENS objective functions are depicted in figures 2 and 3 for the 33-node distribution system with two DGs, respectively. It is clear that the convergence of the proposed algorithm is not a time consuming process since the algorithm converges to the global optima in a few iterations. It is worthwhile to note that the computation time is considered as an important characteristic in power system operation studies.

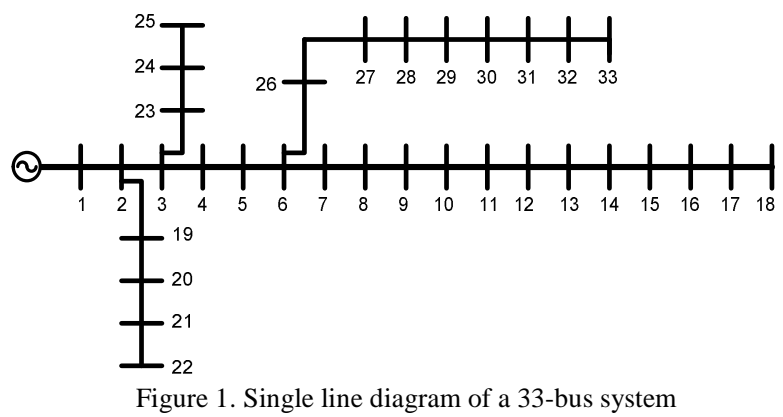


Table I. The obtained results related to section one

\begin{tabular}{|c|c|c|c|c|}
\hline & \multirow{2}{*}{ Base Case } & \multicolumn{2}{|c|}{ Single Objective Cases } & \multirow{2}{*}{ Multi-Objective } \\
\hline & & Loss & ENS & \\
\hline \multirow{2}{*}{$\begin{array}{l}\text { Location of DGs } \\
\text { (Bus No.) }\end{array}$} & - & Bus 12 & Bus 18 & Bus 15 \\
\hline & - & Bus 30 & Bus 33 & Bus 32 \\
\hline \multirow{2}{*}{ Output of DGs (MW) } & - & 1000.00 & 1000.00 & 1000.00 \\
\hline & - & 1000.00 & 1000.00 & 999.00 \\
\hline Loss $(k W)$ & 202.69 & 86.29 & 106.93 & 91.94 \\
\hline ENS (kWh/yr) & 8781.50 & 2876.30 & 2125.50 & 2292.27 \\
\hline
\end{tabular}

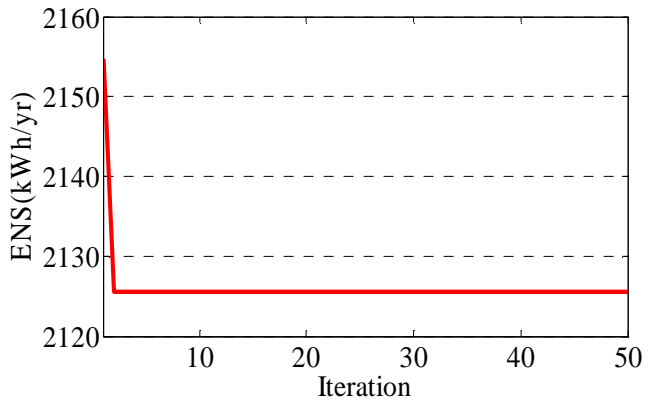

Figure 2. Convergence plot for ENS objective function related to section one

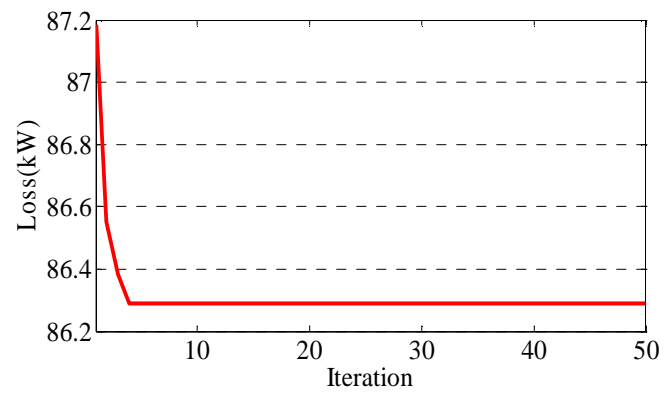

Figure 3. Convergence plot for loss objective function related to section one

According to table I the sizes of DGs are set to their maximum bounds in both single optimizations. So, loss and ENS objectives might be improved more by increasing the DG penetration. Therefore in the following section four DGs have been utilized.

\section{B. Section two: 33-node test system with four DGs}

This test system consists of four DGs. It should be noted that all DGs have a 1000 MW capacity. Like previous section obtained results for loss and ENS objective functions have been shown in table II. From this table it is clear that the power loss and ENS values are decreased drastically by adding DGs. Also, the convergence plot for loss and ENS objective functions are depicted in figures 4 and 5 for 33-node distribution system with four DGs, respectively. Like previous section the proposed algorithm can find the global optima in a few iterations.
Table II. The obtained results related to section two

\begin{tabular}{|c|c|c|c|c|}
\hline & \multirow{2}{*}{ Base Case } & \multicolumn{2}{|c|}{ Single Objective Cases } & \multirow{2}{*}{ Multi-Objective } \\
\hline & & Loss & ENS & \\
\hline \multirow{4}{*}{$\begin{array}{l}\text { Location of DGs } \\
\text { (Bus No.) }\end{array}$} & - & Bus 07 & Bus 18 & Bus 07 \\
\hline & - & Bus 14 & Bus 22 & Bus 16 \\
\hline & - & Bus 24 & Bus 25 & Bus 25 \\
\hline & - & Bus 31 & Bus 33 & Bus 32 \\
\hline \multirow{4}{*}{$\begin{array}{l}\text { Output of DGs } \\
\text { (MW) }\end{array}$} & - & 916.23 & 1000.00 & 1000.00 \\
\hline & - & 585.30 & 1000.00 & 677.17 \\
\hline & - & 980.91 & 1000.00 & 1000.00 \\
\hline & - & 708.55 & 1000.00 & 861.27 \\
\hline Loss $(\mathbf{k W})$ & 202.69 & 65.94 & 102.89 & 71.11 \\
\hline ENS $(\mathbf{k W h} / \mathbf{y r})$ & 8781.50 & 1260.30 & 238.88 & 530.76 \\
\hline
\end{tabular}

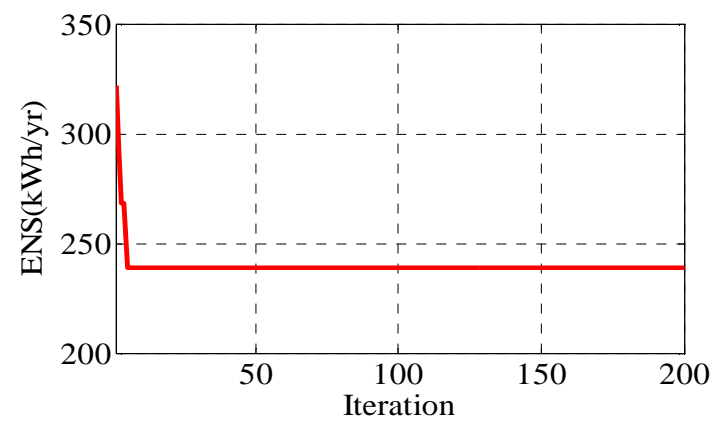

Figure 4. Convergence plot for ENS objective function related to section two

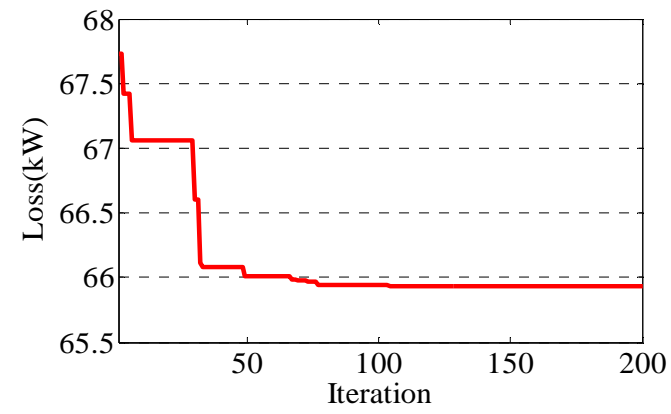

Figure 5. Convergence plot for loss objective function related to section two

\section{Section three : Optimization of different objective functions simultaneously:}

To demonstrate the ability of the presented algorithm in solving complex MOPs Pareto fronts are illustrated in this section. In this regard, the best compromise solutions and their related control variables are depicted in third column of tables I and II. It is noteworthy to say that the best compromise solutions are attained by applying equation (16). Also, it is good to know that the importance factor of different objective functions can be altered in accordance with the system operator decision. In this regard, if one objective is more important than others, the system operator can handle this by increasing the related importance factor of the considered 


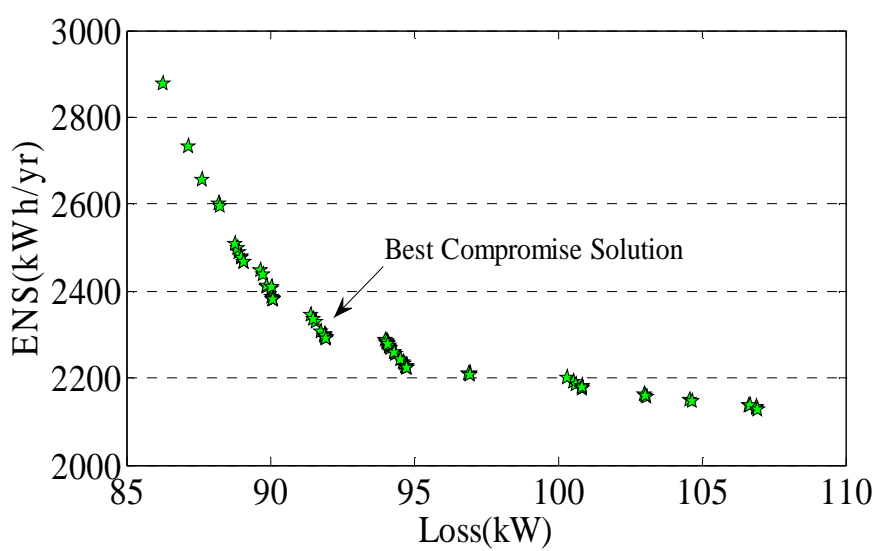

Figure 6. Pareto front for 33-node distribution system with two DGs related to section three

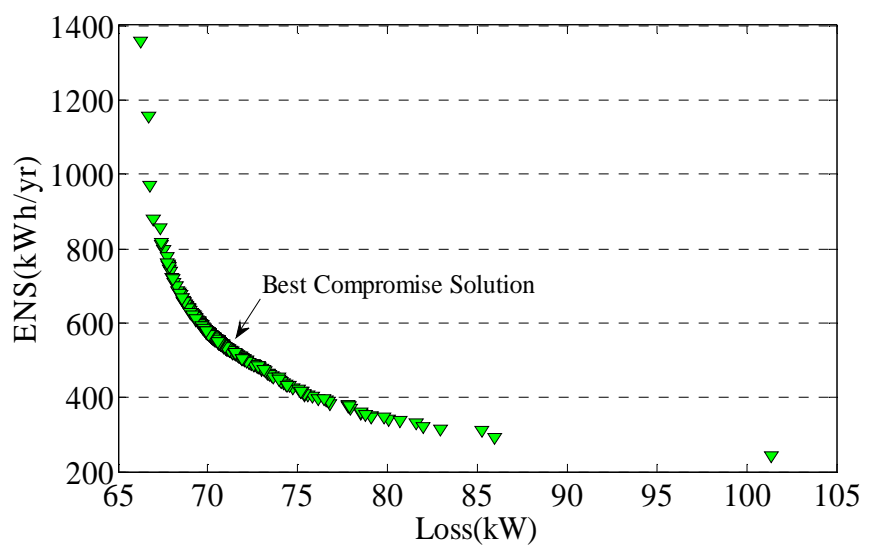

Figure 7. Pareto front for 33-node distribution system with four DGs related to section three

objective function. Therefore, this process assists the system operator to have extensive alternatives. The best operation loss and ENS values in obtained Pareto-optimal solutions are very close to their optimized values, although these objectives are optimized individually, yet the truth of the above mentioned statement is clear in both Pareto fronts. All the above features are provided by applying the proposed algorithm, because fusion of PSO and SFLA increases the search ability of these algorithms.

\section{CONCLUSION}

The optimal sizing and sitting of the DG units in the distribution networks have been studied in this paper using a strong evolutionary algorithm which combines PSO and SFLA methods. Simulation results prove the significant effect of DG units on improvement of distribution network characteristics. The objective functions of the proposed framework are the power losses and the ENS. It is noteworthy to say that the assumed constraints comprising the radial structure of the network, thermal limits of lines, bus voltages and transformer capacities are all within their allowable ranges. This approach has been successfully investigated on the well-known 33 nodes distribution network. Distribution systems would be in a more reliable and economic condition by consideration of ENS and loss objective functions.

\section{REFERENCES}

[1] Mohammad Rasoul Narimani, Ali Azizi Vahed, Rasoul AzizipanahAbarghooee, Mahshid Javidsharifi, Enhanced gravitational search algorithm for multi-objective distribution feeder reconfiguration considering reliability, loss and operational cost, IET Generation. Transmission \&Distribiution, 2013, 1-15.

[2] Mohammad Rasoul Narimani, Rasoul Azizipanah-Abarghooee, Behrouz Zoghdar-Moghadam-Shahrekohne and kayvan gholami, A Novel approach to multi-objective optimal power flow by a new hybrid optimization algorithm considering generator constraints and multi-fuel type, 49, 1 January 2013, 119-136

[3] Zhu D, Broadwater RP, Tam K, Seguin R, Asgeirsson H. Impact of DG placement on reliability and efficiency with time-varying loads. IEEE Transactions on Power Systems2006;21(1):419-27.

[4] Keane A, O'Malley M. Optimal distributed generation plant mix with novel loss adjustment factors. In: Proceedings of IEEE power engineers' society general meeting: 2006.

[5] Moradi MH, Abedini M. A combination of genetic algorithm and particle Swarm optimization for optimal DG location and sizing in distribution systems. International Journal of Electrical Power and Energy Systems 2012;34:66-74.

[6] Niknam T, Taheri SI, Aghaei J, Tabatabaei S, Nayeripour M. A modified honey bee mating optimization algorithm for multi-objective placement of renewable energy resources.Applied Energy2011;88:481730 .

[7] Chiou, J.P., Chang, C.F., Su, C.T.: 'Variable scaling hybrid differential evolution for solving network reconfiguration of distribution systems', IEEE Trans. Power Syst., 2005, 20, (2), pp. 668-674.

[8] Endreneyi, J.: 'Reliability Modeling in Electric Power Systems', NewYork, Wiley, 1978.

[9] Taher Niknam, Mohammad Rasoul Narimani, and Rasoul AzizipanahAbarghooee, A Multi-objective Fuzzy Adaptive PSO Algorithm for Location of Automatic Voltage Regulators in Radial Distribution Networks International Journal of Control, Automation, and Systems (2012) 10(4):1-6. 